\title{
Moving Towards Functional Renal Bioprinting
}

\author{
Emily Thomas $^{\#}$ and Evan Mettenbrink ${ }^{\#}$ \\ Stephenson School of Biomedical Engineering, University of Oklahoma, Norman OK 73071
}

\begin{abstract}
3D bioprinting technologies are rapidly developing and provide a platform for manufacturing structures that mimic the in vivo environment. Recent research aims to produce $3 \mathrm{D}$ bioprinted structures that recapitulate both in vivo structure and functionality. Advancements in both producing high fidelity and functional structures pave the way for full organ bioprinting. Full organ bioprinting holds promise for patients facing renal diseases given both the limited availability of donor kidneys for transplantation which offers the highest quality of life for patients facing renal failure. While the generation of a fully functional bioprinted kidney is a long-term goal, the first step is generating bioprinted functional renal tissue. Functional bioprinted renal tissue may pave the way for full scale organ printing and may offer a more accurate in vitro model for testing the renal toxicity of newly developed therapeutics which holds promise given the limitations of current preclinical in vivo and in vitro models to accurately predict renal toxicity of newly developed therapeutics in humans. Recent work showcases advancements toward renal bioprinting and advancements in the field of bioprinting more broadly may provide opportunities for advancement in renal bioprinting. This review aims to cover recent advances in renal bioprinting and opportunities for innovation. The review seeks to address the mechanical, biological and translational aspects of bioprinting functional renal tissue through an overview of recent advancements (last 5 years) in developing bioinks, utilizing existing 3D bioprinting methods to produce high fidelity printed structures, supporting viability, cell adhesion, cell distribution, functionality and vascularization, and considering important translational aspects of renal bioprinting including larger-scale printing, clinical potential, and prospects towards whole organ generation.
\end{abstract}




\section{Introduction}

3D bioprinting involves the deposition of cell-laden bioinks to create 3-dimensional structures that replicate or mimic in vivo physiology. Although the advent of this technology has long been advertised as a solution to countless problems in regenerative medicine, the present-day state of bioprinting is far from generating patient-specific, functional organs. Regardless, the repetitive functional unit of the kidney makes it an attractive target for bioprinting, and there is a strong need for the development of functional renal tissue. One potential need is in organ transplantation. As of October 2020 , over $83 \%$ of the 119,327 patients on the national organ transplant waitlist were awaiting a kidney transplant ${ }^{1}$. The average person placed on this list will wait over 3.5 years for a kidney transplant, requiring further treatment through dialysis and pharmaceuticals, and 13 of these people waiting for a transplant will die every day ${ }^{2}$. Kidney disease kills more Americans each year than prostate cancer or breast cancer ${ }^{3}$. These numbers are expected to increase as the general population ages ${ }^{4}$. In addition to the lack of available donors, transplantation introduces complications in donor matching, shipping organs, and tissue preservation. Bioprinting entire or portions of kidneys could be an alternative method to organ donation for filling transplant needs. Another potential solution to this problem is prevention of kidney disease through bettering the current technique for determining drug renal toxicity. 3D bioprinting models using human cells more accurately mimic in vivo anatomy and physiology, providing a better screening platform for potential therapeutics before clinical trials begin. This could lead to a reduction in the $19-25 \%$ of acute kidney injury in critically ill patients caused by drug nephrotoxicity ${ }^{5}$. This number has been reported to be as high as $66 \%$ in elderly individuals ${ }^{6}$. A few common examples are: prerenal azotemia, the most common form of kidney failure in hospitalized people, can be caused by diuretics or vasodilators; immunosuppressants and anticoagulants have been linked to renal vascular injury; and various tubular injuries have been associated with antibiotics, chemotherapeutics, and other common clinical compounds ${ }^{7}$. In addition to saving countless lives, better renal toxicity models have the potential to drastically reduce the $\$ 10$ billion spent annually in the United States on phase III drug trial human testing ${ }^{8}$. Nearly half of these phase III trials fail, and of those that fail, 
renal toxicity accounts for nearly $20 \%{ }^{9}$. Improved renal toxicity models could improve in vitro drug testing, preventing more nephrotoxic compounds from reaching late stage trials. Currently, in vitro renal toxicity tests primarily focus on quantifying cell death of primary cultures or in animal models. However, these techniques are often inaccurate at predicting in vivo toxicity ${ }^{9,10}$. Why is bioprinting a promising solution to these problems? Alternative tissue engineering techniques such as electrospinning and soft lithography are not reliably reproducible, unable to accurately create anatomical microstructures like glomeruli, and homogeneous distribution of cells within scaffolds is more difficult to achieve. Although attempts have been made to address these concerns, bioprinting through additive manufacturing is a more promising technique and has been used to reproducibly create multi-material constructs with high resolutions and homogeneous cell distributions at a relatively rapid rate $^{11}$. The attractive properties of bioprinters have led to numerous advancements in tissue engineering. Techniques have been developed enabling printing of multiple materials simultaneously ${ }^{12}$, homogeneously distributed-cell-laden constructs with high viability ${ }^{13}$, and anatomies from medical images ${ }^{14}$. Bioprinting has created implantable stents ${ }^{14}$, bone scaffolds ${ }^{15,16}$, skin tissue $^{16,17}$, and neural cell-integrated muscle tissue ${ }^{18}$. Although the generation of full functional organs is a long-term goal, considering current advances in bioprinting, we propose a short-term goal of printing functional renal tissue. Functional renal tissue would mirror nephron structure; properly mimic the essential kidney functions of filtration, reabsorption, and secretion; and properly alter these functions in response to stimuli such as hypertension. This review serves to aim future research in the right direction to reach the goal of bioprinting functional renal tissue by comprehensively summarizing current literature on renal bioprinting and analyzing prominent literature in related fields with potential application towards the bioprinting of functional renal tissue.

\section{Background}

\subsection{Kidney Physiology}

The repetitive nature of nephrons in kidney anatomy combined with the gradual loss of functionality experienced with kidney disease makes it an attractive organ for bioprinting ${ }^{19}$. Kidney function is 
oftentimes measured by glomerular filtration rate (GFR), which is the amount of blood that is filtered by the kidneys and can be calculated by multiplying the total number of nephrons in a kidney by the GFR of an individual nephron (GFR--nephron * \# of functional nephrons ..= $\left.\mathrm{GFR}_{\text {kidney }}\right)^{20}$. This indicates a linear relationship between kidney GFR and number of functional nephrons in the kidney. Studies have also found linear relationships between age and \# of functional nephrons in a kidney as well as between number of functional nephrons and the progression of chronic kidney disease $\mathrm{e}^{19,21,22}$. These losses of nephrons are accompanied with a corresponding reduction in $\mathrm{GFR}^{21,22}$. Once nephron loss reaches a critical amount, damage is irreversible and is then classified as end-stage renal disease, or kidney failure ${ }^{23}$. This linear relationship enables for the quantitative determination of kidney disease progression by measuring an individual's GFR. Physiologically this means that the biological role of the kidney is equally divided between each of its $\sim 860,000$ nephrons $^{22}$. This contrasts the physiology of other organs, such as the brain, heart, or intestines where functional unit physiology relies on where in the organ the unit resides. For example, an increase in blood pressure caused by increased water reabsorption in the kidney would be caused by a coordinated effort of each individual nephron increasing water reabsorption. This is critical in the pathophysiology of kidney disease as well as in the potential for renal bioprinting. Following an initial renal injury, a small portion of nephrons are lost, which then increases the necessary filtration rates and blood pressure of other nephrons. This induces nephron hypertrophy characterized by an increase in the size of glomerulus, Bowman's capsule, and proximal tubule ${ }^{20}$. This size increase may be potentially harmful and can damage the layer of podocyte cells between the glomerulus and Bowman's capsule which control blood filtration causing detachment, sclerosis of glomerular walls, and eventually nephron atrophy ${ }^{24-26}$. Continued nephron death further increases hypertrophy in remaining functional nephrons, which are then themselves at an increased risk to continue this cycle, further reducing global kidney function. The continuous mechanism of nephron-loss based reduction in kidney function may also point towards promise in bioprinting of renal tissue. Early identification and location could lead to partially replacing kidneys during early stages of chronic kidney disease, preventing disease progression and reducing the nearly $\$ 1$ trillion annually spent on end stage renal disease worldwide ${ }^{28}$. If detected in later stages, upon development 
of a method to reliably print functional renal tissue, bioprinters could be used to customize an implant to replace any section of damaged kidney tissue to restore healthy kidney function and prevent progression to end-stage renal disease. For more information on the specific pathophysiology of chronic kidney disease, or early stages of chronic kidney disease, these two reviews are excellent resources ${ }^{20,27}$.

\subsection{Existing Clinical Options}

The development of a bioprinted kidney would be advantageous since current clinical options fall short. Current clinical options for treating chronic kidney disease include transplant, hemodialysis, and peritoneal dialysis. Additionally, recent research has focused on producing wearable artificial kidney devices which perform dialysis functions in mobile unit. Deceased or living donors may provide the kidneys for transplantation. The five-year survival rate of transplant of patients who received deceased donor kidneys was approximately $79 \%$, and the five-year survival rate of patients who received living donor kidneys was approximately $87 \%$ in 2018 based on unadjusted Kaplan-Meir analysis of patients who received a kidney transplant in $2013^{28}$. While transplantation can treat patients with renal failure, the treatment is limited by the availability of donor organs and allograft rejection may result despite tissue typing and immune suppression. Despite these limitations, organ transplantation offers kidney failure patients with the highest quality of life of the existing clinical options. With organ transplantation, a donor kidney takes on the functions of the patient's failed kidney; in contrast, dialysis does not provide a cure and instead is only a management strategy for the chronic disease. While dialysis methods may act as a substitute for native renal filtration and can prolong a patient's life, none of the existing dialysis options restore full native kidney functionality.

One of the earliest developed renal failure treatments is hemodialysis which was first applied successfully in $1945^{29}$ and remains the most common treatment for chronic renal failure globally ${ }^{30}$. In hemodialysis, an external machine filters a patient's blood. The hemodialysis system is based around a dialyzer which separates blood from a dialysate with a semipermeable membrane ${ }^{30}$. Based on the solute composition of the dialysate which can be tuned by the dialysis administrator, small waste solutes will pass from the 
patient's blood across the semipermeable membrane into the dialysate, and the newly filtered blood is returned to the patient. While hemodialysis can effectively filter blood, significant limitations exist. Most patients must travel to a dialysis center three times a week and each session takes approximately four hours $^{31}$. Alternatively, approximately $12 \%$ of hemodialysis patients receive home dialysis. While home dialysis eliminates the need for travel to a center, it requires extensive training and many patients require a caretaker to assist with the dialysis ${ }^{32}$. Furthermore, hemodialysis has poor long-term survival rates. In 2017 the hemodialysis mortality rate was approximately 17\% based on the 2019 United States Renal Data Systems Report ${ }^{33}$.

An alternative to hemodialysis is peritoneal dialysis. In peritoneal dialysis, the patient's peritoneal membrane, the serous lining of the abdominal cavity, is used as a semipermeable membrane instead of a synthetic external semipermeable membrane as in hemodialysis. There are two major types of peritoneal dialysis: continuous ambulatory dialysis (CAPD) and continuous cycling peritoneal dialysis (CCPD). In CAPD a catheter is used to fill a patient's abdominal cavity with dialysate and the dialysis fluid is allowed to dwell in the patient as solute exchange occurs between the patient's blood and the dialysate. The fluid is exchanged between an external dialysate bag, the patient's abdomen, and an external collection bag three to four times during the day and once overnight. As opposed to hemodialysis, CAPD occurs passively during the course of a patient's routine ${ }^{34}$. In CCPD, an automated style of peritoneal dialysis, a device called a cycler automatically fills and drains the patient's abdominal cavity with dialysis fluid. The exchange is performed three to four times overnight and once during the day. In a study of patient opinions of peritoneal dialysis, $94 \%$ of a cohort of 2760 randomly sampled respondents from seven countries between 2014 and 2017 categorized the ability to receive treatment from home as a major advantage ${ }^{35}$. The most frequently listed disadvantages by the cohort were experiencing a bloated feeling during treatment and the storage space required by peritoneal dialysis supplies ${ }^{35}$. Additionally, patients undergoing peritoneal dialysis are at risk of peritonitis, a potentially life-threating infection that often results from contaminated catheter 
insertion $^{36}$. In addition to an increased risk of peritonitis, CCPD-related increases in intra-abdominal pressure cause increased rates of abdominal wall complications such as hernias ${ }^{37}$.

Wearable artificial kidneys (WAK) may potentially offer an improved solution to chronic kidney disease. WAKs are devices that perform hemodialysis, peritoneal dialysis, or a combination of the two techniques in a mobile fashion. A WAK filters a patient's blood with an artificial semipermeable membrane or the patient's peritoneal membrane in a wearable lightweight device, and the WAK also regenerates the dialysate, permitting continuous mobile filtration ${ }^{38}$. Unlike dialysis methods which are common clinical options, no WAKs have received FDA approval to date. One major obstacle to commercially available WAKs is the removable of toxins without the large volumes of dialysate required for traditional dialysis methods. Current research aims to reduce the amount of dialysate required through recycling used dialysate. Dialysate can be regenerated using sorbents, which bindtoxins in the dialysate removing them from the body $^{39}$. Common sorbents include activated charcoal ${ }^{10}$ and zirconium phosphate ${ }^{40,41}$. Additionally, since the most commonly used sorbents fail to adsorb urea, WAKs may incorporate a urease column for the enzymatic elimination of urea ${ }^{4213}$ or rely on electro- ${ }^{43}$ or photo oxidation ${ }^{44}$ to eliminate urea waste.

\subsection{Need for Renal Bioprinting}

There is a definite need to address the limitations of transplants and dialysis methods. Transplantation is limited by donor organ supply and risk of allogenic rejection, and dialysis methods filter patient's blood but are dependent on cumbersome external equipment. Whole organ bioprinting may one day provide patients with substitute kidneys that recapitulate native tissue function. However, additive manufacturing of a full transplantable kidney has not yet been realized. The generation of bioprinted kidneys will require significant advances in large-scale vascularized organ bioprinting. A successfully bioprinted kidney will mimic the mechanical and cellular aspects of the native kidney.

\subsection{Challenges in Whole Organ Bioprinting}


Whole organ bioprinting involves depositing heterogenous cell types and scaffold to create in vivo scale synthetic organs. Several challenges have limited the translational potential of whole organ bioprinting. A transplantable bioprinted kidney will need to possess mechanical and biological characteristics that mimic the native kidney. To address biological considerations the material must support cell adhesion, viability, and native functionality. Mechanical properties are dependent on the selected bioink as well as bioprinting method. Bioinks will need to be developed that provide mechanical support and support biological function. Since organs such as the kidney are heterogeneous in structure, the printing of complex gradients of materials and bioactive cues will be required. However,the field of developing complex heterogeneous bioinks is still its infancy ${ }^{45}$. Additionally, the selected bioprinting method must support high resolution printing to recreate delicate organ structures such as the kidney's tubular network ${ }^{46}$.

Another challenge is sourcing the cells to create a real-size printed organ. The large number of cells that will not illicit an immune required for organ printing often cannot be obtained from autograft alone, and instead in vitro expansion will be required. Primary cell lines have limited expansion potential because higher passage cells may not exhibit native behavior. For example, at greater than three passages, renal tubular cells exhibited senescence ${ }^{47}$.

Solid organs such as the kidney pose unique biological challenges for bioprinting. For example, the translation of small in vitro bioprinted structures to full-size adult organs will require the creation of a vascularized network so that nutrients and wastes can be exchanged deep within the printed construct to avoid cell death at the core ${ }^{48}$. Current advances in inducing or creating vascularized printed tissues that may be leveraged in renal bioprinting include using blend bioinks ${ }^{49}$, incorporating gelatin microparticles to create porous microstructures $^{50}$, incorporating angiogenic growth factors such as vascular endothelial growth factor $^{51,52}$, and microchanneling ${ }^{53}$.

To meet these challenges, full organ kidney bioprinting will require leveraging existing advances made in bioprinting and pioneering methods to address the current limitations in whole-organ additive manufacturing. 
This review serves to aim future research in the right direction to attain the goal of bioprinting functional renal tissue. To do this, the review will cover problems faced in renal bioprinting, and propose different methods to solve them. In writing this review, we will include research on development of the bioinks useful in renal bioprinting and their corresponding print structure; the incorporation of cellular aspects such as viability, adhesion, cell distribution, functionality, and vascularization; and the translational aspects of bioprinting renal tissue including larger-scale printing, clinical potential, and prospects towards whole organ generation. We expect our limits of research to be defined by a comprehensive assessment of all recent (past 5 years) advances in renal bioprinting as well as potentially applicable advances in non-renal bioprinting technology.

\section{Recent Advancements}

\subsection{Printing Structure}

The final printed structure is dependent on the printing method selected and the determines in part the structural fidelity and mechanical performance of the final printed construct. Advances in kidney bioprinting showcase some of the early attempts at solid organ bioprinting which faces a unique set of challenges. A printed construct should support mechanical stresses likely to be placed on the construct when implanted in vivo while maintaining porosity necessary for waste and nutrient perfusion. Three main methods of 3D bioprinting, extrusion-based, laser-based and droplet-based, exist, each with different advantages and disadvantages as we look toward full functional kidney bioprinting. ${ }^{54}$

\subsubsection{Extrusion-based Bioprinting}

Extrusion-based bioprinting involves pressure or a mechanical plunger to extrude a biomaterial. Extrusionbased bioprinting has been used to print a wide variety of biological structures including but not limited to $\operatorname{cardiac}^{55}$, liver ${ }^{56}$, and cartilage ${ }^{57}$ tissue engineering. Advantages of extrusion-based bioprinting include the ability to print porous scaffolds and soft materials. ${ }^{58}$ However, one primary disadvantage of extrusion bioprinting techniques is that lower resolution is possible than can be achieved with other techniques, and 
strategies for increasing resolution such as reducing nozzle size may place additional shear stresses on the cell in the bioink reducing post-printing viability. Higher resolution extrusion-printed structures have been achieved with the freeform reversible embedding of suspended hydrogel (FRESH) bioprinting technique. ${ }^{59}$ With the FRESH technique the soft biomaterial is extruded into a microparticle bath that provides support until the material can be crosslinked into a solid structure. With the FRESH technique resolution has been shown to be a linear function of nozzle diameter with printed resolution ranging from $20 \mu \mathrm{m}$ to $200 \mu \mathrm{m}$ for needles with diameters from $10 \mu \mathrm{m}$ to $200 \mu \mathrm{m}(\mathrm{R} 2=0.97)$. The high resolution achieved with soft biomaterials through FRESH printing is promising for printing high fidelity structures such as kidney nephrons. Since soft biomaterials will likely be used to bioprint kidneys, extrusion-based bioprinting is a promising technique.

\subsubsection{Droplet Based}

Droplet-based bioprinting is a technique that uses inkjet, acoustic-droplet-ejection or micro-valve technology to deposit a series of droplets to form biomimetic structures. ${ }^{60}$ Advantages of droplet-based bioprinting involve potential to produce heterogenous patterns of cells and biological factors. For example, droplet-based bioprinting has been used to print high-resolution interfaces of human embryonic kidney cells and mesenchymal stem cells. ${ }^{60}$ Such advancement paves the way for generating a heterogenous multicellular full-scale bioprinted kidney. However, distinct limitations of droplet-based bioprinting exist. Due to limited range of droplet-based bioprinting suitable bioinks it is difficult to produce large and structurally stable constructs. ${ }^{7}$

\subsection{Laser-based Bioprinting and Stereolithography}

Laser-based bioprinting and stereolithography both utilize light to produce 3D-bioprinted constructs. In laser-based bioprinting, a focused laser is used to direct the deposition of a bioink a droplet-bydroplet fashion. Laser-based bioprinting allows precise printing with a non-contact nozzle free approach. ${ }^{61}$ Laser-based bioprinting has been used to bioprint dermal tissue ${ }^{62}$, cornea-mimicking structures $^{63}$, and many more biological constructs. However, laser-based bioprinting is limited by increased manufacturing time compared to other methods and decreased cell viability in printed constructs. ${ }^{64}$ On the 
other hand, stereolithography involves using a light source to solidify layers of light sensitive materials, building structures in a layer-by-layer fashion. Stereolithography has the highest accuracy of the methods discussed. ${ }^{61}$ Stereolithography is limited by the paucity of biocompatible light sensitive biomaterials as many developed have toxic byproducts and the technique does not permit the formation of horizontal gradients reducing the complexity of materials that can be generated. ${ }^{65}$

\subsection{Current Bioinks Used in Renal Printing}

Full organ bioprinting requires selection of an appropriate bioink. A bioink is a cell-laden biomaterial deposited by the selected printing method. All bioinks used in renal bioprinting must meet three distinct criteria: 1) compatibility with selected printing method 2) desirable post-printing mechanical properties 3) biocompatibility. A fibrinogen and gelatin bioink for 3D printing renal structures has been developed by the Lewis group that meets these criteria. ${ }^{66}$ The fibrinogen bioink was fabricated to include the enzymes thrombin and transglutaminase which permit the solidification of the bioink. The thrombin converts fibrin to fibrinogen while transglutaminase more slowly crosslinks gelatin with fibrinogen to fuse printed layers together. A fugitive ink that liquifies when cooled was used to form hollow tubule structures. This bioink supported renal cell viability and functionality. The Atala group recently developed an methacrylated decellularized kidney ECM-derived bioink, KdECMMA, for printing renal tissue ${ }^{67}$ The bioink possessed key functional properties including a viscosity that prevents cell settling, shear thinning behavior to prevent cell damage during extrusion, and rapid photoinitiated crosslinking postdeposition. Furthermore, the material supported enhanced cell proliferation compared to a gelatin only control $(\mathrm{P}<0.05) .{ }^{67}$ Additionally, a San-Diego biotechnology company, Organovo, working in collaboration with the Murdoch Children's Research Institute, published a preprint showcasing the development of a cell-only paste that can act as a bioink for printing renal organoids. ${ }^{68}$ Figure 1 showcases all reported bionks used at the time of this review used for renal bioprinting. The limited list of bioinks described here reflects the infancy of the field of renal bioprinting. 3D-dimensional renal cell culture is presently a more established field than renal bioprinting and showcases materials that may be adapted for 3D bioprinting. 


\section{Bioink}

A. ECM Bioink
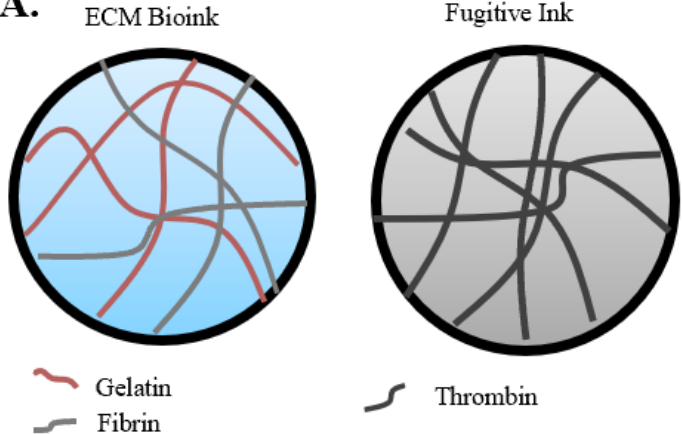

Final Construct

Proximal Tubule Mimic

Perfused with Cells
B.

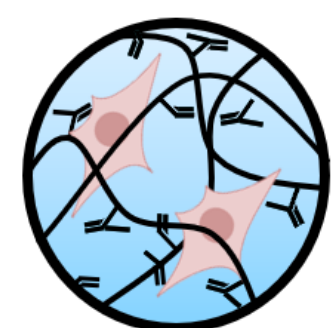

HEK 293 T

KdECMMA

Tubular and

Glomerular

Structures
C.

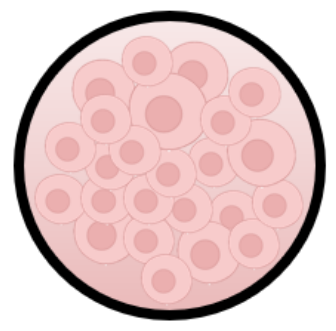

Human Pluripotent

Stem Cell

Kidney Organoid

Figure 1: A. Gelatin / fibrin bioink used to print proximal tubule structures by the Lewis Group. B. kdECMMA bioink used to print tubular and glomerular structures by the Lee group. C. Cell only bioink used to print kidney organoids by Organova.

\subsection{Current Biomaterials Used for 3D Renal Cell Culture and their Potential as Bioinks}

The following referenced papers show promise in 3D renal cell culture and may be aptly suited for bioprinting. Recently a novel heparin-based PEG-star hydrogel was shown to promote human renal tubulogenesis in vitro. Similar multi-armed PEG based hydrogels have been previously bioprinted showing potential of for the heparin-based material as bioink ${ }^{69}$; however, further material characterization would be necessary to determine if the heparin modified material possesses the rheological and post-printing characteristics required for a bioink. Additionally, collagen hydrogels have been shown to support the formation of renal structures. ${ }^{70,71}$ Collagen has served as a bioink for many nonrenal bioprinting applications including to generate high resolution structures using the FRESH bioprinting technique. ${ }^{55}$ Furthermore, many ECM-derived biomaterials have been used to support 3D renal cell culture and could be adapted to serve as bioinks as shown by the Atala group. ${ }^{72,73,74,75}$ See the review here for a more complete overview of bioinks for bioprinting. ${ }^{76}$ 


\subsection{Cell Adhesion}

To maintain functionality of the printed scaffolds before the structures are perfused with media, cell attachment to the printed material must occur. Some naturally derived bioinks promote cell adhesion without modification. For example, Yeong et al showed primary kidney cell adhesion to a decellularized porcine ECM scaffold. ${ }^{77}$ Additional, tissue engineering applications have shown natural renal cell adhesion to ECM-derived biomaterials. ${ }^{67,72-75,77}$ Furthermore, fibrin ${ }^{78}$ and collagen ${ }^{55,71}$ biomaterials have been shown to support renal cell adhesion. If synthetic materials, such as PEG, are used as bioinks, additional factors may need to be added to the materials to promote cell adhesion. A common technique to increase cell adhesion to non-adherent biomaterials such as PEG is modification of the synthetic polymer with cell adhesion promoting peptide sequences such as RGD. ${ }^{79}$

\subsection{Cell Distribution}

3D bioprinting of a full functional kidney will require printing multiple cell types in an organized distribution to mimic native cell arrangement; however, the bioprinting of multiple kidney cell types has not yet been realized. A functional kidney construct will feature a complex arrangement of cell types as reflected by kidney histology shown in Figure 1. 


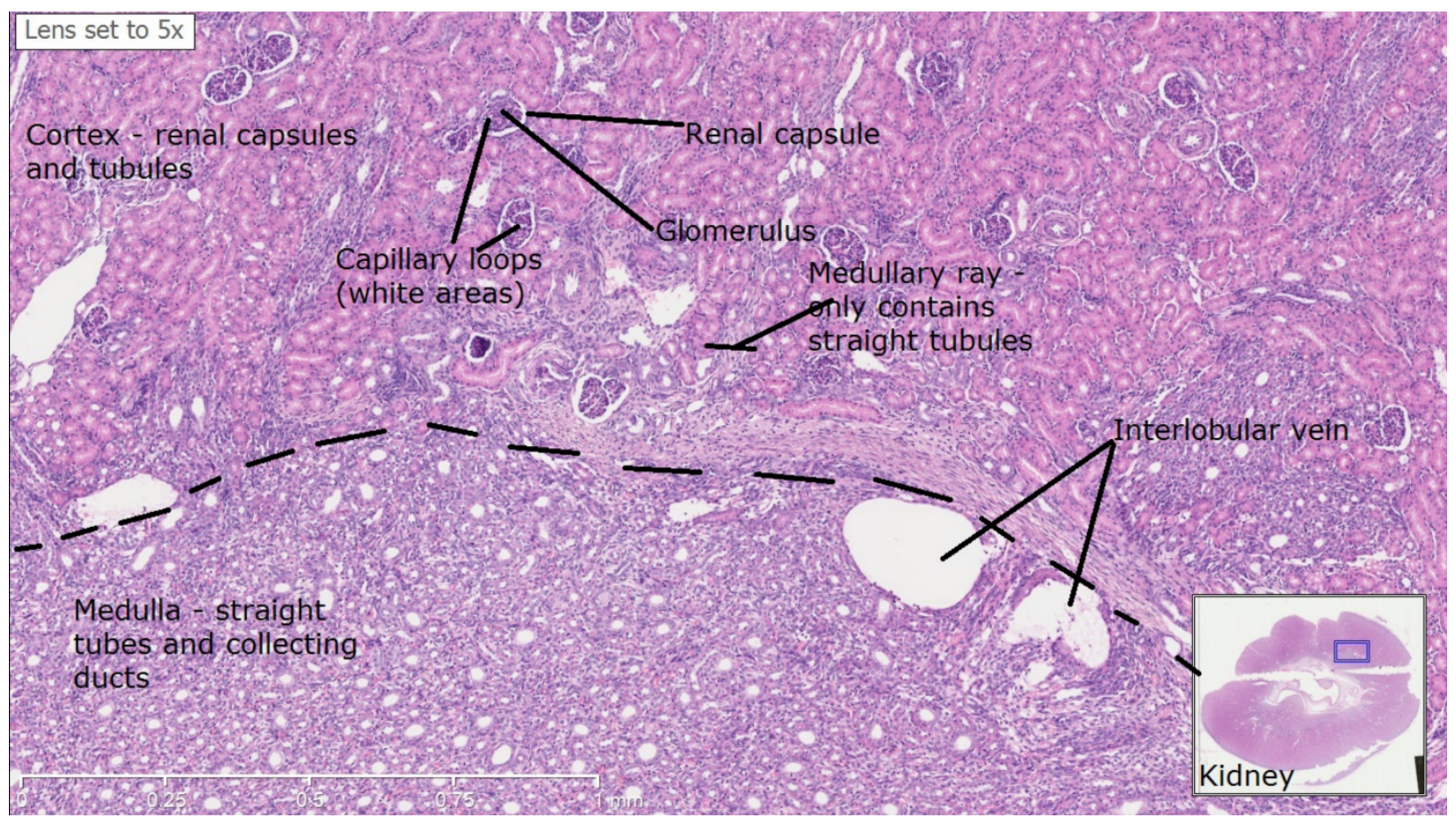

Figure 1: Hematoxylin and eosin staining of the kidney shows the complex arrangement of heterogenous cell types forming renal structures including the glomerulus, cortex and medulla. (Image from University of Nottingham. ${ }^{80}$ )

While multiple cell types have not been printed for renal tissue engineering. Current advances in bioprinting showcase heterogenous cell type bioprinting for applications such as cardiac and urethra tissue engineering where similar techniques may one day be applied to printing renal structures. For example, multicellular heart tissue has been bioprinted by using multiple microfluidic pumps. ${ }^{81}$ Additionally, multicellular urethra tissue was printed using the integrated organ printing technique (IOP). ${ }^{82}$ IOP manufacturing uses multiple pressure-controlled cartridges with each connected to a microscale nozzle. ${ }^{83}$ Furthermore, Liu et al demonstrated that it was possible to fabricate constructs with up to four cell types using a novel multimaterial extrusion bioprinting platform. ${ }^{84}$

\subsection{Cell Viability}

In addition to creating a print structure with high resolution and print fidelity, bioprinted structures must also be able to sustain cell viability throughout the entirety of the bioprinting process and during subsequent culture. During the printing process, shear stresses and extensional stresses caused by needle extrusion 
of bioinks are the primary cause of decreases in cell viability. ${ }^{85}$ Factors that decrease stresses such as increasing the print nozzle diameter, decreasing bioink viscosity, and decreasing print pressure have been found to increase cell viability post-print. ${ }^{86,87,88}$ The opposite effect is found in print fidelity and accuracy. When nozzle size increases, pressure decreases, and viscosity decreases, print accuracy and fidelity decreases ${ }^{87}$ Nair et. al. developed models to predict the proportions of live, injured and dead cells as a function of both pressure and nozzle diameter. ${ }^{86}$ Other groups have measured or created models predicting cell viability of different cell lines based on quantifications of shear stresses or extension stresses in bioprinting. ${ }^{85,88}$ A promising method to achieving relatively high print fidelity while also maintaining strong cell viability is FRESH bioprinting. ${ }^{59}$ This method suspends bioinks of interest in a secondary hydrogel bath, enabling printing of low-viscosity shear-thinning hydrogels with high accuracy. Following the printing of the bioinks, due to the 3-dimensional structure of renal tissue, ample nutrient supply to all portions of the structure is critical for maintaining high cell viability throughout culture and tissue development. Without ample nutrient delivery, cell viability, specifically in the center of the scaffold, may decrease due to hypoxia-induced apoptosis. ${ }^{89,90}$ Within renal bioprinting, the supply of growth factors to induce proper differentiation of stem cells is also crucial in future creation of anatomically and physiologically accurate tissue. A promising approach to short-term nutrient supply to cells throughout a bioprinted scaffold was summarized in a recent paper where a Ge1MA gel is printed simultaneously with a gelatin sacrificial gel. Upon photo-crosslinking the Ge1MA gel, the gelatin is washed away, allowing for nutrient diffusion throughout the gel and high cell viability throughout the bioprinted structure. ${ }^{91}$ Ouyang et al. optimized print parameters for HEK293 cells and achieved nearly 95\% post-extrusion viability at room temperature, long-term stability, and normal biological functions of these cells by printing a macroporous structure. ${ }^{92}$ However, it was noted that shear-stresses caused a reduction in cell viability and beyond one month cell viability decreased. The authors hypothesized this was due to lack of proper nutrient diffusion. ${ }^{9}$ Potentially by combining these optimal print properties with the sacrificial gel through FRESH printing, the cell viability could be even further improved as well as an increase in structural integrity and long-term nutrient diffusion. It is also worth noting that cell viability has been shown to increase when 
cultured in three-dimensions or when renal tubule cells polarize and participate in vascular-endothelial cross-talk characteristic of nephrons. ${ }^{93,94}$

\subsection{Functionality}

To date, most attempts at recapitulating nephron physiology in vitro aim at creating a functional proximal tubule. This is because the proximal tubule is the most damaged site in kidney injury, making it a target for nephrotoxicity studies, as well as being the site of $65-80 \%$ of nutrient reabsorption into the bloodstream. ${ }^{66}$ Typical proximal tubule function is characterized by uptake across the apical membrane and exit from the cell across the basolateral membrane. ${ }^{95}$ The capability of membrane transport is visible in the polarization of human kidney cells, where certain organelles and transporters localize to either the basolateral membrane or the apical membrane depending on their function. Specifically, mitochondria tend to be more heavily concentrated on the basolateral side of the cell where golgi bodies tend to gather on the apical side of the cell. ${ }^{96}$ Primary attempts to artificially mimic renal tissue function begin here, and these morphological characteristics in addition to responses to nephrotoxins, hyperglycemic or hypersaline conditions are oftentimes used to quantify the success of such attempts. Numerous hydrogels have been found that induce tubulogenesis or renal function in vitro. It was found that a photo-crosslinkable porcine ECM hydrogel combined with gelatin, hyaluronic acid, and glycerol for rheological properties contributed to elevated viability, polarity, and amino acid transfer in a non-structured hydrogel ${ }^{67}$ Furthermore, the solubilized ECM induced immunohistochemical-analyzed tubulogenesis significantly better than a gelatin methacrylate gel. ${ }^{67}$ Similar successes were also observed with a synthetic four-armed maleimide polyethylene glycol hydrogel with optimized conditions, providing an alternative to xenogenic ECM hydrogels. ${ }^{97}$ Another study found that a heparin-based hydrogel induced tubulogenesis with consistent morphology and architecture to native tissue. These cells also showed organic ion transfer and proper response to nephrotoxins. ${ }^{96}$ Many attempts have been made to mimic proximal tubule function in vitro without bioprinting in order to better nephrotoxicity testing in clinical trials. ${ }^{93,94}$ By creating adjacent linear microchannels, vascular-endothelial cross-talk was able to be replicated. They found that the polarization and biomarker expression of human kidney cells increased in a 3D culture as well as their 
viability. ${ }^{93}$ Also, sodium-coupled glucose transport, albumin uptake, and intracellular enzyme function was replicated in addition to significantly higher cell count when kidney cells were cultured adjacent to endothelial cells $(p<0.001) .{ }^{93,94}$ Although these systems are able to recapitulate functionality of the proximal tubule, the ability to create larger functional tissues or organs is limited due to the inability to customize curvature, size, and shape of the nephron portions due to the fabrication technique. Considering these limitations, bioprinting is a convenient solution to this issue, as similar techniques can be used to create customizable tubular architectures. The Lewis lab at Harvard University has been able to successfully combine the successes in microfluidic systems and the advantageous nature of bioprinting to rapidly print functional proximal tubule architectures for cell seeding. ${ }^{66,98}$ The process involves the deposition of ECMderived hydrogels over a sacrificial gel printed to the custom shape of the proximal tubule. After dissolution of the sacrificial gel, PTEC-TERT1 cells are able to be cultured in the microchannels to confluency. The initial tests showed this model, similarly to the other microfluidic systems, was able to properly model cell polarization as well as albumin uptake and response to nephrotoxins. These tubules were viable for the entirety of the 65 -day tests. ${ }^{66}$ In subsequent publications, vascular channels were able to be printed adjacently to tubular channels, enabling albumin uptake as well as glucose reabsorption based on external signals. ${ }^{98}$ This bioprinting method shows promise towards the fabrication of larger quantities of proximal tubules and accompanying vasculature. Beyond immediate function, innervation may be a difficult task in functional renal tissue generation. In muscle tissue, it was found that mixing neural stem cells with the bioprinted muscle progenitor cells in a 1:300 ratio increased muscular generation, long-term viability, and was able to generate more force than the muscle cell only model 8 weeks after implantation $(p=0.047) .{ }^{99}$ Similar techniques could be applied to renal tissue, enhancing function, integration into the host nervous system, and long-term cell viability of tissue structures.

\subsection{Vasculature}

As previously stated, a primary hurdle faced in larger scale tissue engineering is sustaining long-term nutrient delivery and waste elimination through functional vascular networks. Without this development, living bioprinted tissue has a limit of a few hundred micrometers in thickness. ${ }^{100}$ Although microchannels 
are able to support cell viability immediately after printing, as printed cells proliferate and differentiate, secreted ECM fills the hydrogel, hindering the ability for microchannels to ensure nutrient delivery inside the scaffold. In a similar fashion to functional proximal tubules, bioprinting of vascularized tissue typically involves the printing of multiple hydrogels, followed by the washing away of a sacrificial gel, leaving microchannels where vascular endothelial cells can be cultured. Commonly used sacrificial gels include Pluronic F-127, carbohydrate-glass, gelatin, and agarose. ${ }^{101}$ Early developments and printing of microchannels occurred used FRESH printing and similar methods. ${ }^{102,59}$ Hinton and colleagues used FRESH to print an MRI-derived coronary artery system with $<15 \%$ variability in dimensions. ${ }^{59}$ Approximately the same time, Bhattacharjee et al. used Carbopol hydrogels and local injections to reach similar accomplishments, printing hierarchically branched networks with diameters in the 100um range was able to seed cells into these microchannels. ${ }^{102}$ With more general applications, sacrificial writing into functional tissues (SWIFT) printing suspends grouped iPSC's, termed embryonic bodies and organ building blocks, into ECM and Matrigel hydrogels before using bioprinters to inject sacrificial ink into microchannel locations of interest. Using this technique, a microchannel was developed, and media was used to differentiate iPSC's into cardiomyocytes, which developed a synchronous beat following 7 days of culture. The authors do note cell confluency was difficult to achieve. This technique could be applied to differentiate autologous iPSC's into epithelial and renal cells to create sustainable vascularized renal tissue. ${ }^{19}$ The Lewis lab was also able to achieve similar results in osteogenic tissue engineering. Multi-material bioprinting was used to create cylindrical vessels with elastic walls printed into a mesenchymal stem cell and neonatal dermal fibroblast hydrogel. Confluency was observed and viability was sustained for 6 weeks. Thick tissues were developed by creating multiple vessels, which were able to maintain cell viability throughout $1 \mathrm{~cm}$ thick tissues. ${ }^{103}$ Attempting to demonstrate the accuracy of their microchannel printing method, Grigoryan et al used tartrazine food dye in a photopolymerizing gel to bioprint functioning alveolar vasculature. Oxygen transport was observed in this model by quantifying blood oxygen level before and after alveolar interaction. Most notably, as alveolus and glomerular sizes are roughly equivalent, similar techniques could be adapted to model glomerular filtration in vitro. ${ }^{104} \mathrm{~A}$ final approach to printing vasculature in tissue 
constructs is the use of coaxial printing, where vascular endothelial cells are printed through the same nozzle as tissue-specific cells with a sacrificial gel. This approach eliminated the need for cell perfusion into the scaffold and instead showed strong confluency of cells in vessels by simply perfusing tailored media into the scaffold. Furthermore, heterogeneous structures with multiple cell types are able to be printed through the same print nozzle, enabling the printing of heterogeneous cell structures. This method was applied with success to both osteogenic and cancer tissue, however relatively low cell viability was achieved with only $\sim 80 \%$ of cells being viable following printing and after culture. ${ }^{105}$ It has been shown that large-scale vascular networks can be developed, challenges moving forward include development of blood vessels with sub 100um diameters, integrating tissue function and vasculature, and maintaining high cell viability in more developed tissue constructs.

\subsection{Towards Large-Scale Tissue Bioprinting}

As many of the studies highlighted in this review focus on bioprinting of structures $<1 \mathrm{~cm}$ in length, significant advances in bioprinting must occur before whole-organ printing is realized. ${ }^{103}$ Although one work estimated the ability of their technique to be upscaled to nearly $100 \mathrm{~mL}$ hydrogels, at this scale, the sourcing of such large quantities of, in that specific case, iPSCs is another obstacle to overcome in itself. ${ }^{100}$ Furthermore, as scaffolds grow in size, cell viability may also decrease. ${ }^{105}$ If taking the approach established by Lin et al where sacrificial inks are utilized for tubule development, ${ }^{98}$ preserving accurate tubule structures throughout the extensive time frame printing would occur followed by preservation of channels and accurate placements of heterogeneous cell types using perfusion are all much more difficult to obtain on a larger scale. Coaxial printing may hold the key to printing heterogeneous cell types but maintaining cell viability during printing as opposed to perfusion may prove challenging. However, promising progress has been shown in printing vascular channels and upscaling this process may overcome problems in nutrient supply and waste removal. With regards to future manufacturing, customizable kidney morphologies for implantation would require methods to create personal CAD models, which would require MRI scans to be converted to printable files with both accurate structures and cell types. A method may need to be developed to accomplish this conversion from medical imaging to printable files. In short, 
print fidelity and structure upon upscaling of these methods and accurate culture and deposition of heterogeneous cell types at a scale which is magnitudes larger than currently shown are major challenges that need to be addressed.

\subsection{Renal Bioprinting in Clinical Applications}

To achieve the bioprinting of transplantable kidneys in a clinical setting, added challenges include maintaining structure and viability during transportation of tissue following biofabrication; integration into the patient's nervous, circulatory, and excretory system; and ensuring long-term function and stability postimplantation. Transportation may be solved through supplying surgical hospitals with bioprinters or through implementing similar techniques to retain tissue viability currently used in transplantation. In vivo studies of muscle implants in murine models successfully accomplished integration with host nervous systems through mixing low concentrations of neural stem cells into bioinks. ${ }^{99}$ For vascular integration, a technology termed the AngioChip accomplished anastomosis in rat models using connection through surgical cuffs, observing native angiogenesis a week following implantation. ${ }^{106}$ Similar surgical methods could be used to attach the much larger renal collecting duct to ureters for waste excretion. Finally, longterm stability and immunogenicity in the patient are critical for developing medical technologies that meet standard of care. To achieve this, autologous iPSCs could be used to prevent the ethical considerations and variability of other stem cells as well as reducing transplant immunogenicity as cells would be sourced from the patient. This approach was used by Skylar-Scott and colleagues while bioprinting of vasculature using the SWIFT method. ${ }^{100}$ Additionally, to print larger full organ structures it will be critical to amplify suitable, ideally autologous, cell sources to produce sufficient scaffold cell confluency. For more complete guidance on use stem cells in tissue engineering we direct readers to the review here. ${ }^{107}$

\section{Conclusion}

In this text, recent research on renal bioprinting is discussed. Rapid advancements are being made towards being able to print three-dimensional renal tissue. Both synthetically and naturally derived bioinks have been developed with the ability to mimic natural kidney mechanical properties as well as 
achieve high cell viabilities, stimulate cell adhesion, and maintain shape fidelity. Furthermore, bioprinting techniques have achieved the resolution required to accurately replicate renal tubular architectures including glomeruli, tubules, and corresponding blood vessels. The ability to print these structures has been combined with cell-seeding techniques in order to create functioning blood vessels, recapitulating proximal tubule-arteriole cross talk, showing the ability to alter absorption in response to external stimuli. These models can better predict nephrotoxicity than simple culture of compounds of interest with kidney cells. Furthermore, techniques utilizing sacrificial gels have printed complex vascular networks, enabling the bioprinting of anatomically relevant thick tissues. Beginning work has shown promise in neural, tubular, and vascular host integration as well. Despite these advances, sizable progress is required to realize bioprinting as a platform for creating functional renal tissue. First, a primary need in renal bioprinting research is the ability to source, culture, and properly print large enough cell quantities to create tissue structures capable of implant. Secondly, although individual proximal tubules have been developed, and alveolar structures could match glomerular structures, the bioprinting of a single nephron with all functional parts has not yet been achieved, this could be the next step in bioprinting fully functional tissue. A potential obstacle in this process using existing techniques is the differentiation in physiology between proximal and distal tubules. In addition to creating entire nephrons, functional tissue would require multiple nephrons working together to filter blood and collect filtrate for urine production. To the author's knowledge, no attempt has been made at multiple nephron bioprinting. With the upscale of the number of nephrons being printed, more accurate cell seeding techniques may be needed in order to selectively coat different tubes with the correct cell types. Furthermore, fully recreating renal function would require differentiation between juxtamedullary and cortical nephrons and their functions. This would also require more accurate cell-seeding and differentiation techniques than currently accomplished. Multi-material bioprinting may hold the key to unlocking this potential, and the ability to seed regionspecific growth factors into hydrogels before the addition of renal or vascular cells could enable the induction of accurate regional physiologies at the nephron and the kidney level. Ultimately, advances in bioprinting technology will pave the way for functional renal tissue printing. 


\section{Authorship}

${ }^{\#}$ Emily Thomas and Evan Mettenbrink contributed equally

References

1. National Data - OPTN. https://optn.transplant.hrsa.gov/data/view-data-reports/national-data/ (2020).

2. Organ Donation and Transplantation Statistics. National Kidney Foundation https://www.kidney.org/news/newsroom/factsheets/Organ-Donation-and-Transplantation-Stats (2014).

3. Kidney Disease Statistics for the United States | NIDDK. National Institute of Diabetes and Digestive and Kidney Diseases https://www.niddk.nih.gov/health-information/health-statistics/kidney-disease.

4. NASRI, H. World Kidney Day 2014; Chronic Kidney Disease and Aging: A Global Health Alert. Iran. J. Public Health 43, 126-127 (2014).

5. Bicalho, M. D., Soares, D. B., Botoni, F. A., Reis, A. M. M. \& Martins, M. A. P. Drug-Induced Nephrotoxicity and Dose Adjustment Recommendations: Agreement Among Four Drug Information Sources. Int. J. Environ. Res. Public. Health 12, 11227-11240 (2015).

6. Kim, S. Y. \& Moon, A. Drug-Induced Nephrotoxicity and Its Biomarkers. Biomol. Ther. 20, 268-272 (2012).

7. Choudhury, D. \& Ahmed, Z. Drug-associated renal dysfunction and injury. Nat. Clin. Pract. Nephrol. 2, 80-91 (2006).

8. Fogel, D. B. Factors associated with clinical trials that fail and opportunities for improving the likelihood of success: A review. Contemp. Clin. Trials Commun. 11, 156-164 (2018).

9. Homan, K. A. et al. Bioprinting of 3D Convoluted Renal Proximal Tubules on Perfusable Chips. Sci. Rep. 6, 34845 (2016).

10. Faria, J., Ahmed, S., Gerritsen, K. G. F., Mihaila, S. M. \& Masereeuw, R. Kidney-based in vitro models for drug-induced toxicity testing. Arch. Toxicol. 93, 3397-3418 (2019). 
11. Chandra, P. K., Soker, S. \& Atala, A. Chapter 1 - Tissue engineering: current status and future perspectives. in Principles of Tissue Engineering (Fifth Edition) (eds. Lanza, R., Langer, R., Vacanti, J. P. \& Atala, A.) 1-35 (Academic Press, 2020). doi:10.1016/B978-0-12-818422-6.00004-6.

12. Liu, W. et al. Rapid Continuous Multimaterial Extrusion Bioprinting. Adv. Mater. 29, 1604630 (2017).

13. Kesti, M. et al. Bioprinting Complex Cartilaginous Structures with Clinically Compliant Biomaterials. Adv. Funct. Mater. 25, 7406-7417 (2015).

14. Da, Z., Sj, H., Me, N., Rg, O. \& Ge, G. Bioresorbable airway splint created with a threedimensional printer. The New England journal of medicine vol. 368

https://pubmed.ncbi.nlm.nih.gov/23697530/ (2013).

15. Zhai, X. et al. 3D-Printed High Strength Bioactive Supramolecular Polymer/Clay Nanocomposite Hydrogel Scaffold for Bone Regeneration. ACS Biomater. Sci. Eng. 3, 1109-1118 (2017).

16. F, Z. et al. Rapid printing of bio-inspired 3D tissue constructs for skin regeneration. Biomaterials vol. 258 https://pubmed.ncbi.nlm.nih.gov/32847683/ (2020).

17. Albanna, M. et al. In Situ Bioprinting of Autologous Skin Cells Accelerates Wound Healing of Extensive Excisional Full-Thickness Wounds. Sci. Rep. 9, 1856 (2019).

18. Kim, J. H. et al. Neural cell integration into 3D bioprinted skeletal muscle constructs accelerates restoration of muscle function. Nat. Commun. 11, 1025 (2020).

19. Schnaper, H. W. Remnant nephron physiology and the progression of chronic kidney disease. Pediatr. Nephrol. Berl. Ger. 29, (2014).

20. Romagnani, P. et al. Chronic kidney disease. Nat. Rev. Dis. Primer 3, 1-24 (2017).

21. Denic, A. et al. The Substantial Loss of Nephrons in Healthy Human Kidneys with Aging. J. Am. Soc. Nephrol. 28, 313-320 (2017).

22. Denic, A. et al. Single-Nephron Glomerular Filtration Rate in Healthy Adults. N. Engl. J. Med. 376, 2349-2357 (2017). 
23. Schaefer, B. \& Wühl, E. Educational paper: Progression in chronic kidney disease and prevention strategies. Eur. J. Pediatr. 171, 1579-1588 (2012).

24. D'Agati, V. D. et al. Obesity-related glomerulopathy: clinical and pathologic characteristics and pathogenesis. Nat. Rev. Nephrol. 12, 453-471 (2016).

25. Kriz, W. \& Lemley, K. V. A potential role for mechanical forces in the detachment of podocytes and the progression of CKD. J. Am. Soc. Nephrol. JASN 26, 258-269 (2015).

26. Fukuda, A. et al. Growth-Dependent Podocyte Failure Causes Glomerulosclerosis. J. Am. Soc. Nephrol. JASN 23, 1351-1363 (2012).

27. Wouters, O. J., O’Donoghue, D. J., Ritchie, J., Kanavos, P. G. \& Narva, A. S. Early chronic kidney disease: diagnosis, management and models of care. Nat. Rev. Nephrol. 11, 491-502 (2015).

28. Hart, A. et al. OPTN/SRTR 2018 Annual Data Report: Kidney. Am. J. Transplant. 20, 20-130 (2020).

29. Gottschalk, C. W. \& Fellner, S. K. History of the Science of Dialysis. Am. J. Nephrol. 17, 289298 (1997).

30. Kotanko, P., Kuhlmann, M. K. \& Levin, N. W. CHAPTER 89 - Hemodialysis: Principles and Techniques. in Comprehensive Clinical Nephrology (Fourth Edition) (eds. Floege, J., Johnson, R. J. \& Feehally, J.) 1053-1059 (Mosby, 2010). doi:10.1016/B978-0-323-05876-6.00089-7.

31. What is Dialysis? National Kidney Foundation https://www.kidney.org/atoz/content/dialysisinfo (2015).

32. Masterson, R. The advantages and disadvantages of home hemodialysis. Hemodial. Int. 12, S16S20 (2008).

33. 2020 U.S. organ and tissue transplants. 24 (2020).

34. Vardhan, A. \& Hutchison, A. J. 59 - Peritoneal Dialysis. in National Kidney Foundation Primer on Kidney Diseases (Sixth Edition) (eds. Gilbert, S. J. \& Weiner, D. E.) 520-533 (W.B. Saunders, 2014). doi:10.1016/B978-1-4557-4617-0.00059-5. 
35. Sukul, N. et al. Patient-reported advantages and disadvantages of peritoneal dialysis: results from the PDOPPS. BMC Nephrol. 20, 116 (2019).

36. Salzer, W. L. Peritoneal dialysis-related peritonitis: challenges and solutions. Int. J. Nephrol. Renov. Dis. 11, 173-186 (2018).

37. Del Peso, G. et al. Risk Factors for Abdominal Wall Complications in Peritoneal Dialysis Patients. Perit. Dial. Int. 23, 249-254 (2003).

38. Bazaev, N. A., Putrya, B. M., Zhilo, N. M. \& Grinval'd, V. M. Wearable Artificial Kidney Design Principles. in 2018 IEEE East-West Design Test Symposium (EWDTS) 1-6 (2018). doi:10.1109/EWDTS.2018.8524761.

39. Agar, J. W. Review: Understanding sorbent dialysis systems. Nephrology 15, 406-411 (2010).

40. Roberts, M. The regenerative dialysis (REDY) sorbent system. Nephrology 4, 275-278 (1998).

41. Wong, R. J.-H. Acid zirconium phosphate and alkaline hydrous zirconium oxide materials for sorbent dialysis. (2013).

42. Gerber, M. T. \& Hobot, C. M. Replenishing urease in dialysis systems using a urease introducer. (2018).

43. Wester, M. et al. Removal of Urea in a Wearable Dialysis Device: A Reappraisal of ElectroOxidation. Artif. Organs 38, 998-1006 (2014).

44. Hinds, B. \& Shao, G. Apparatus and method for urea photo-oxidation. (2020).

45. Murphy, S. V., De Coppi, P. \& Atala, A. Opportunities and challenges of translational 3D bioprinting. Nat. Biomed. Eng. 4, 370-380 (2020).

46. Costantini, F. \& Kopan, R. Patterning a Complex Organ: Branching Morphogenesis and Nephron Segmentation in Kidney Development. Dev. Cell 18, 698-712 (2010).

47. Luttropp, D., Schade, M., Baer, P. C. \& Bereiter-Hahn, J. Respiration rate in human primary renal proximal and early distal tubular cells in vitro: Considerations for biohybrid renal devices. Biotechnol. Prog. 27, 262-268 (2011). 
48. Jafarkhani, M., Salehi, Z., Aidun, A. \& Shokrgozar, M. A. Bioprinting in Vascularization

Strategies. Iran. Biomed. J. 23, 9-20 (2019).

49. Jia, W. et al. Direct 3D bioprinting of perfusable vascular constructs using a blend bioink.

Biomaterials 106, 58-68 (2016).

50. Lee, A. et al. 3D bioprinting of collagen to rebuild components of the human heart. Science $\mathbf{3 6 5}$, 482-487 (2019).

51. des Rieux, A. et al. 3D systems delivering VEGF to promote angiogenesis for tissue engineering. J. Controlled Release 150, 272-278 (2011).

52. Nourse Marilyn B. et al. VEGF Induces Differentiation of Functional Endothelium From Human Embryonic Stem Cells. Arterioscler. Thromb. Vasc. Biol. 30, 80-89 (2010).

53. Stosich, M. S. et al. Vascularized Adipose Tissue Grafts from Human Mesenchymal Stem Cells with Bioactive Cues and Microchannel Conduits. Tissue Eng. 13, 2881-2890 (2007).

54. Cho, D.-W. et al. 3D Bioprinting Techniques. in 3D Bioprinting: Modeling In Vitro Tissues and Organs Using Tissue-Specific Bioinks (eds. Cho, D.-W. et al.) 25-29 (Springer International Publishing, 2019). doi:10.1007/978-3-030-32222-9_4.

55. Lee, A. et al. 3D bioprinting of collagen to rebuild components of the human heart. Science $\mathbf{3 6 5}$, 482-487 (2019).

56. Billiet, T., Gevaert, E., De Schryver, T., Cornelissen, M. \& Dubruel, P. The 3D printing of gelatin methacrylamide cell-laden tissue-engineered constructs with high cell viability. Biomaterials $\mathbf{3 5}$, 4962 (2014).

57. You, F., Eames, B. F. \& Chen, X. Application of Extrusion-Based Hydrogel Bioprinting for Cartilage Tissue Engineering. Int. J. Mol. Sci. 18, 1597 (2017).

58. Ozbolat, I. T. \& Hospodiuk, M. Current advances and future perspectives in extrusion-based bioprinting. Biomaterials 76, 321-343 (2016).

59. Hinton, T. J. et al. Three-dimensional printing of complex biological structures by freeform reversible embedding of suspended hydrogels. Sci. $A d v$. 1, e1500758 (2015). 
60. Gudapati, H., Dey, M. \& Ozbolat, I. A comprehensive review on droplet-based bioprinting: Past, present and future. Biomaterials 102, 20-42 (2016).

61. Li, J., Chen, M., Fan, X. \& Zhou, H. Recent advances in bioprinting techniques: approaches, applications and future prospects. J. Transl. Med. 14, 271 (2016).

62. Michael, S. et al. Tissue engineered skin substitutes created by laser-assisted bioprinting form skin-like structures in the dorsal skin fold chamber in mice. PloS One 8, e57741 (2013).

63. Sorkio, A. et al. Human stem cell based corneal tissue mimicking structures using laser-assisted 3D bioprinting and functional bioinks. Biomaterials 171, 57-71 (2018).

64. Bishop, E. S. et al. 3-D bioprinting technologies in tissue engineering and regenerative medicine: Current and future trends. Genes Dis. 4, 185-195 (2017).

65. Melchels, F. P. W., Feijen, J. \& Grijpma, D. W. A review on stereolithography and its applications in biomedical engineering. Biomaterials 31, 6121-6130 (2010).

66. Homan, K. A. et al. Bioprinting of 3D Convoluted Renal Proximal Tubules on Perfusable Chips. Sci. Rep. 6, 34845 (2016).

67. Ali, M. et al. A Photo-Crosslinkable Kidney ECM-Derived Bioink Accelerates Renal Tissue Formation. Adv. Healthc. Mater. 8, e1800992 (2019).

68. Higgins, J. W. et al. Bioprinted pluripotent stem cell-derived kidney organoids provide opportunities for high content screening. bioRxiv 505396 (2018) doi:10.1101/505396.

69. Skardal, A., Zhang, J. \& Prestwich, G. D. Bioprinting vessel-like constructs using hyaluronan hydrogels crosslinked with tetrahedral polyethylene glycol tetracrylates. Biomaterials 31, 6173-6181 (2010).

70. Joraku, A., Stern, K. A., Atala, A. \& Yoo, J. J. In vitro generation of three-dimensional renal structures. Methods 47, 129-133 (2009).

71. Shen, C., Zhang, G., Wang, Q. \& Meng, Q. Fabrication of Collagen Gel Hollow Fibers by Covalent Cross-Linking for Construction of Bioengineering Renal Tubules. ACS Appl. Mater. Interfaces 7, 19789-19797 (2015). 
72. Su, J., Satchell, S. C., Shah, R. N. \& Wertheim, J. A. Kidney Decellularized Extracellular Matrix Hydrogels: Rheological Characterization and Human Glomerular Endothelial Cell Response to Encapsulation. J. Biomed. Mater. Res. A 106, 2448-2462 (2018).

73. Rj, N. et al. Decellularized Human Kidney Cortex Hydrogels Enhance Kidney Microvascular Endothelial Cell Maturation and Quiescence. Tissue Eng. Part A 22, 1140-1150 (2016).

74. O’Neill, J. D., Freytes, D. O., Anandappa, A. J., Oliver, J. A. \& Vunjak-Novakovic, G. V. The regulation of growth and metabolism of kidney stem cells with regional specificity using extracellular matrix derived from kidney. Biomaterials 34, 9830-9841 (2013).

75. Hiraki, H. L., Nagao, R. J., Himmelfarb, J. \& Zheng, Y. Fabricating a Kidney Cortex Extracellular Matrix-Derived Hydrogel. JoVE J. Vis. Exp. e58314 (2018) doi:10.3791/58314.

76. Mobaraki, M., Ghaffari, M., Yazdanpanah, A., Luo, Y. \& Mills, D. K. Bioinks and bioprinting: A focused review. Bioprinting 18, e00080 (2020).

77. Chae, S. Y. et al. Development of renal extracellular matrix (ECM) scaffold for kidney regeneration. Tissue Eng. Regen. Med. 11, 1-7 (2014).

78. Ng, C. P., Zhuang, Y., Lin, A. W. H. \& Teo, J. C. M. A Fibrin-Based Tissue-Engineered Renal Proximal Tubule for Bioartificial Kidney Devices: Development, Characterization and In Vitro Transport Study. International Journal of Tissue Engineering vol. 2013 e319476 https://www.hindawi.com/journals/ijte/2013/319476/ (2012).

79. Hersel, U., Dahmen, C. \& Kessler, H. RGD modified polymers: biomaterials for stimulated cell adhesion and beyond. Biomaterials 24, 4385-4415 (2003).

80. Porcine Kidney Histology | OVAM. http://www.onlineveterinaryanatomy.net/content/porcinekidney-histology.

81. Maiullari, F. et al. A multi-cellular 3D bioprinting approach for vascularized heart tissue engineering based on HUVECs and iPSC-derived cardiomyocytes. Sci. Rep. 8, 13532 (2018). 
82. Zhang, K. et al. 3D bioprinting of urethra with PCL/PLCL blend and dual autologous cells in fibrin hydrogel: An in vitro evaluation of biomimetic mechanical property and cell growth environment. Acta Biomater. 50, 154-164 (2017).

83. Kang, H.-W. et al. A 3D bioprinting system to produce human-scale tissue constructs with structural integrity. Nat. Biotechnol. 34, 312-319 (2016).

84. Liu, W. et al. Rapid Continuous Multimaterial Extrusion Bioprinting. Adv. Mater. 29, 1604630 (2017).

85. Ning, L., Betancourt, N., Schreyer, D. J. \& Chen, X. Characterization of Cell Damage and Proliferative Ability during and after Bioprinting. ACS Biomater. Sci. Eng. 4, 3906-3918 (2018).

86. Nair, K. et al. Characterization of cell viability during bioprinting processes. Biotechnol. J. 4, 1168-1177 (2009).

87. Schwab, A. et al. Printability and Shape Fidelity of Bioinks in 3D Bioprinting. Chem. Rev. (2020) doi:10.1021/acs.chemrev.0c00084.

88. Ouyang, L., Yao, R., Zhao, Y. \& Sun, W. Effect of bioink properties on printability and cell viability for 3D bioplotting of embryonic stem cells. Biofabrication 8, 035020 (2016).

89. Berg, J. et al. Optimization of cell-laden bioinks for 3D bioprinting and efficient infection with influenza A virus. Sci. Rep. 8, 13877 (2018).

90. Kesti, M. et al. Bioprinting Complex Cartilaginous Structures with Clinically Compliant Biomaterials. Adv. Funct. Mater. 25, 7406-7417 (2015).

91. Shao, L. et al. Synchronous 3D Bioprinting of Large-Scale Cell-Laden Constructs with Nutrient Networks. Adv. Healthc. Mater. 9, 1901142 (2020).

92. Ouyang, L., Yao, R., Chen, X., Na, J. \& Sun, W. 3D printing of HEK 293FT cell-laden hydrogel into macroporous constructs with high cell viability and normal biological functions. Biofabrication 7, 015010 (2015).

93. Weber, E. J. et al. Development of a microphysiological model of human kidney proximal tubule function. Kidney Int. 90, 627-637 (2016). 
94. Vedula, E. M., Alonso, J. L., Arnaout, M. A. \& Charest, J. L. A microfluidic renal proximal tubule with active reabsorptive function. PLOS ONE 12, (2017).

95. Jenkinson, S. E. et al. The limitations of renal epithelial cell line HK-2 as a model of drug transporter expression and function in the proximal tubule. Pflüg. Arch. - Eur. J. Physiol. 464, 601$611(2012)$

96. Weber, H. M., Tsurkan, M. V., Magno, V., Freudenberg, U. \& Werner, C. Heparin-based hydrogels induce human renal tubulogenesis in vitro. Acta Biomater. 57, 59-69 (2017).

97. Cruz-Acuña, R., Mulero-Russe, A., Clark, A. Y., Zent, R. \& García, A. J. Identification of matrix physicochemical properties required for renal epithelial cell tubulogenesis by using synthetic hydrogels. J. Cell Sci. 132, (2019).

98. Lin, N. Y. C. et al. Renal reabsorption in 3D vascularized proximal tubule models. Proc. Natl. Acad. Sci. 116, 5399-5404 (2019).

99. Kim, J. H. et al. Neural cell integration into 3D bioprinted skeletal muscle constructs accelerates restoration of muscle function. Nat. Commun. 11, 1025 (2020).

100. Skylar-Scott, M. A. et al. Biomanufacturing of organ-specific tissues with high cellular density and embedded vascular channels. Sci. Adv. 5, (2019).

101. Bova, L., Billi, F. \& Cimetta, E. Mini-review: advances in 3D bioprinting of vascularized constructs. Biol. Direct 15, 22 (2020).

102. Bhattacharjee, T. et al. Writing in the granular gel medium. Sci. Adv. 1, e1500655 (2015).

103. Kolesky, D. B., Homan, K. A., Skylar-Scott, M. A. \& Lewis, J. A. Three-dimensional bioprinting of thick vascularized tissues. Proc. Natl. Acad. Sci. 113, 3179-3184 (2016).

104. Grigoryan, B. et al. Multivascular networks and functional intravascular topologies within biocompatible hydrogels. Science 364, 458-464 (2019).

105. Shao, L. et al. Directly coaxial 3D bioprinting of large-scale vascularized tissue constructs. Biofabrication 12, 035014 (2020). 
106. Zhang, B. et al. Biodegradable scaffold with built-in vasculature for organ-on-a-chip engineering and direct surgical anastomosis. Nat. Mater. 15, 669-678 (2016).

107. Concise Review: Bioprinting of Stem Cells for Transplantable Tissue Fabrication - PubMed. https://pubmed.ncbi.nlm.nih.gov/28836738/. 